Books, videos, cd-roms, dvds and any other relevant items submitted for a review in the BDJ should be addressed to:

Mike Grace, Editor, British Dental Journal,

64 Wimpole Street W1G 8YS

\section{Human disease for dentists}

\author{
Ed. D.J. Gawkrodger \\ Oxford: Blackwell, 2004 \\ price $€ 29.99$, pp296 \\ ISBN 0632064536
}

This textbook is 280 pages long with simple line drawings and a colour plate section. It is easily readable for dental undergraduates, at whom it is chiefly directed. Ambitiously, it has a very wide scope with six sections covering basic skills, dealing with: history and examination; diseases and conditions covering systemic medicine; and others varying from cardiovascular disease, to allergy, to pregnancy, to disability. There is a section on pharmacology and anaesthetics including general anaesthesia followed by revision and answer sections. Section 6 is dedicated to the now archaic practice of putting colour plates together at one end of the book.

The scope of this text is so wide that the explanations are, by necessity, often very short and perhaps difficult for the novice to grasp. Many explanations which would clarify individual sections have been curtailed. The relevance to dentistry is prominent in some sections such as asthma, but is patchy in others: virtually no specific information relevant to dentistry appears in the section on haemophilia, and the advice on INR levels is at variance with nationally accepted guidelines. Of the 35 contributors to the text, only one is a dentist, and this keeps the text less in touch with contemporary thinking relating to dental management of the medically compromised. For example, the need to cover dental procedures with additional steroids in patients taking corticosteroid medication is no longer a widely held view within the UK.

Regardless, this text is admirably readable, covers a wide scope of material relevant to the management of medically compromised patients in a dental setting, and is likely to be a popular undergraduate text.

\section{Atlas of oral and maxillofacial rehabilitation}

\begin{tabular}{l}
\hline Ed. K. Seto with M. Matsuura, K. Shimozato, M. \\
Goto and T. Nomura \\
Surrey: Quintessence \\
price $£ 48$, pp 112 \\
ISBN 4874177964
\end{tabular}

This multi-authored densely illustrated practical manual on prosthetic rehabilitation for maxillary and mandibular defects provides a well written basis for indications, classification of defects, design and manufacture of maxillofacial prostheses.

Section 1 highlights the classification of maxillofacial prostheses, the simulation of the defects and physiological aspects of rehabilitation (speech, eating and swallowing). The subsequent sections include chapters that specifically address maxillary defects, palatal and oropharyngeal defects, mandibular defects, facial defects and radiation stents. Each chapter illustrates the classification of different defects, highlighting the potential problems for rehabilitation. Also included in each section is a well illustrated step by step account of the laboratory techniques. There are often useful tips on evaluating access to the available oral working space (section 2) and tables that lay out the relative advantages and disadvantages of available materials. There is minimal discussion on the use of implants in relation to these defects (included in Chapters 10 and 18) and this perhaps reflects the relative novelty of implant use in these patients.

Despite being a multi-authored book, there is a consistent logical approach to each defect with minimal repetition providing an excellent 'whistle stop tour' of the world of maxillofacial prostheses. The illustrations are mainly excellent and overall this book is a very useful practical manual and should be a useful companion to surgical trainees, prosthodontists and dental technicians alike.

T.F. Renton

\section{Selection criteria for dental radiography}

\author{
Ed. M.E. Pendlebury, K. Horner, K. Eaton \\ UK: The Faculty of General Dental Practitioners \\ price £30, pp 108 \\ ISBN 0954345118
}

This is the second edition of the only UK publication to look at selection criteria for dental radiography in all disciplines of dentistry. The review editors have successfully updated the contents where possible and modernised the appearance.

The introduction is well written and assists the reader in understanding the remainder of the publication, in particular the methodology and review process that was used. The inclusion of papers as evidence follows the SIGN methodology, and papers with an unacceptable level of bias were excluded.

The editors state 'The main value of selection criteria is in reducing the collective dose to the population by eliminating unnecessary or unproductive radiographic examination'; there is repeated reference to the importance of a thorough history and examination prior to any radiographic examination.

A number of sections have been expanded and new ones introduced. Within each section re-ordering has resulted in the more critical points being covered earlier. The section on panoramic radiography has been expanded and concludes with an important message that "there is no justification for review panoramic radiography at arbitrary time intervals' This is reinforced in the Good Practice section by a 'Don't: Carry out “screening” radiographs or take radiographs as "routine”. A new section on application of digital radiography gives a clear introduction to the available technological options, and makes it clear that selection criteria is essentially no different than for film radiography. Reference to digital and other diagnostic methods also occurs within the section dealing with dental caries diagnosis. The section on radiographs in implantology has been expanded and includes reference 

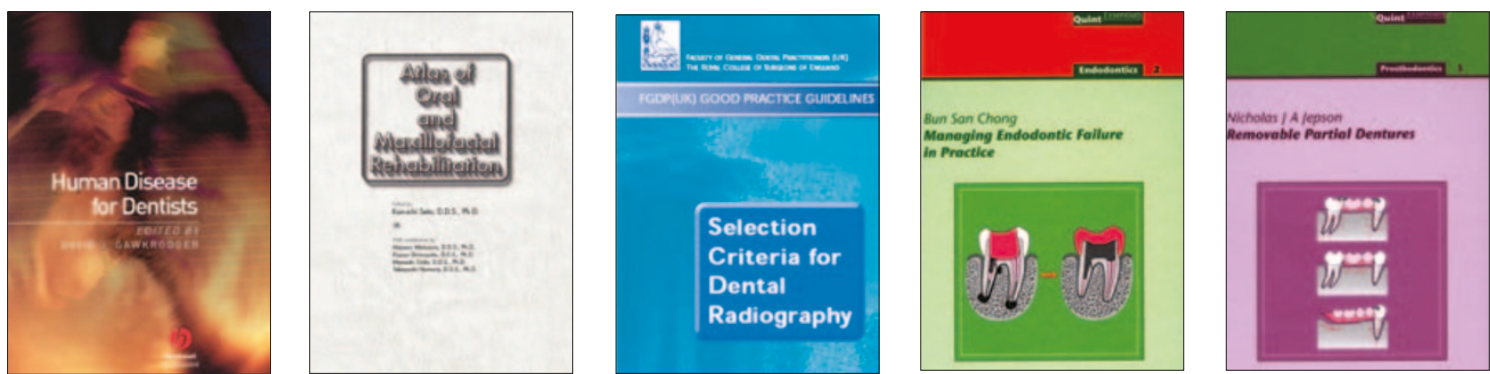

to the relatively recent use of magnetic resonance imaging (MRI) as an option for some patients. A summary table detailing the appropriate options for implant treatment planning is included.

The index is greatly expanded, giving a good overview that improves the ease of finding specific items, and is followed by a helpful 'recommendations at a glance'.

When the first edition was published (1998), it was clear that there was a lack of strong evidence for many of the recommendations. Whilst it must have been hoped that further research would produce this evidence, there is, unfortunately, little change. The editors highlight the gaps in section 1.10, providing a useful list for future research.

Overall, this new edition is welcome and a helpful reference for educators, those studying for higher qualifications, and practitioners in all branches of dentistry.

L.M. Brocklebank

\section{Managing endodontic failure in practice}

\author{
Ed. Bun San Chong \\ Surrey: Quintessence \\ price $\mathrm{\epsilon} 28, \mathrm{pp} 152$ \\ ISBN 1850970866
}

This book is excellent! It is one of a series written specifically for the general dental practitioner and I cannot see how anyone who enjoys the daily challenge of dental practice could not be enthralled by the contents. The author is an acknowledged expert in endodontic treatment, but he approaches this subject from a truly honest and practical perspective, in an easy-to read style. This is not a stodgy theoretical textbook; every dentist will learn something from almost every page, and enjoy the process as well!

In the first three chapters the author considers realistically the concept of success and failure, but then challenges the reader to decide whether or not retreatment is actually indicated, necessary, possible, or
- in particular - within the operator's skill. I found this section immensely valuable, and will refer to it widely in my own practice and teaching.

Having re-confirmed the biological aims of endodontic treatment in general, and retreatment in particular, the reader is then led by the hand through the various clinical procedures necessary to disassemble the coronal restoration, access blocked canals, remove separated instruments and other obstructions. However, while guiding the practitioner through these various procedures the reader is encouraged to consider the possible problems and pitfalls, and to question his or her own skills and experience.

This book will be an invaluable asset to all those faced with the problems of endodontic failure for two reasons. First it will give significant help and guidance in making a diagnosis of the cause of failure and the possible treatment options, and second it will then focus the practitioner's thoughts in an honest appraisal of the skills and techniques required to carry out the correct treatment which may, of course, be referral to an expert. The author is certainly one of those!

\section{Removable partial dentures}

\author{
Ed. N.J.A. Jepson \\ Surrey: Quintessence, 2004 \\ price $€ 28$, pp 148 \\ ISBN 1850970750
}

This book, designed to cover the fundamentals of removable partial dentures (RPDs) for general practitioners more than achieves its goal. The sensible, no nonsense approach combined with the excellent reproduction of both the clinical photography and illustrations should make the book a first choice text for dental undergraduates. Its small size belies the wealth of informed comment within.

Well written, the book deals with the dogma attached to partial denture design theory and proposes a straightforward step- by-step approach. I was particularly impressed with the first two chapters dealing with the rationale behind the provision of RPDs and more importantly 'not providing' a prosthesis. Dealing with the shortened dental arch concept early in a text of this kind is a welcome change of direction. It is particularly encouraging to see topics such as indirect retention and guide planes well explained and treated with a little caution. The goal of this text is to promote the use of simple functional designs, rather than a slavish reliance on mechanics.

The chapters titled 'Clinical Guides' are particularly good and well illustrated. The chapter dealing with acrylic-resin transitional partial dentures is very good. UK prosthodontists are particularly strong at applying the principles of biologically sound design to such prostheses. This is something that the foremost transatlantic textbooks should improve upon. I would have preferred a little less emphasis on the temporary nature of these prostheses. Admittedly many such dentures end up with immediate additions in poorly maintained mouths. However, there really is no sound evidence to support the idea that an acrylic-resin mucosa-supported denture would inevitably damage a wellmaintained and regularly monitored mouth.

There are some minor glitches with the illustrations in this first edition. I am sure that figure 4.4a does not show what the caption hopes it does. The prosthesis in figure $4.2 \mathrm{~b}$ has such a good appearance that it is difficult to discern what is artificial and what is natural tooth, therefore its impact is lost. Perhaps labels identifying the components would help? Similarly figure $3.12 \mathrm{~b}$ is a little obscure. In addition, postgraduate students may find the further reading and referencing a little sparse. To be honest this is nit picking and would not stop me recommending this text.

I would therefore recommend this book as an excellent tool for CPD for the dental practitioner and a strong reference text for undergraduates. 